\title{
REPRESENTACIONES RUPESTRES TARDÍAS EN MORRITOS, SAN ANTONIO DE LOS COBRES, PUNA DE SALTA. OBSERVACIONES E HIPÓTESIS PRELIMINARES.
}

\author{
Hernán Juan Muscio \\ Instituto de Arqueología, FFyL, UBA-Conicet \\ 25 de mayo, 217, CABA, (1002), hmuscio@fibertel.com.ar \\ Presentado el: 30/06/2010 - Aceptado 15/07/2010
}

\section{Introducción}

En esta contribución presento, sucintamente, algunos aspectos de las representaciones rupestres del sitio Morritos Alero 2 del Período Tardío. El sitio pertenece a un conjunto de sitios localizados en el paraje Los Patos, cuenca de San Antonio de los Cobres, Salta, Argentina (Figura 1). El conjunto se dispone sobre la parte superior de una terraza aluvial elevada por encima de los $200 \mathrm{~m}$ sobre el cauce del río Los Patos, cuya altitud es de 3900 $\mathrm{msnm}$, y con una pendiente abrupta. En la cumbre de esta geoforma la evidencia arqueológica se distribuye heterogéneamente en una superficie escarpada y sin disponibilidad de agua. Por su ubicación en lo alto, desde Morritos se domina visualmente el valle de San Antonio de los Cobres y las Salinas Grandes hacia el Norte y hacia el sureste el Acay y la planicie que lo circunda. Entre la evidencia arqueológica hay estructuras arquitectónicas aisladas y agrupadas. Las estructuras aisladas son de planta circular que varían entre los 12 y 26 m de diámetro. Por su emplazamiento y tamaño se trataría de corrales, mientras que las pocas estructuras agrupadas corresponderían a recintos, ya que sus dimensiones no superan los 4 $\mathrm{m}$ de diámetro y sus plantas son de forma irregular, también localizadas en estos sectores acantilados, que además presentan aleros naturales cuya excavación reportó evidencia arqueológica diversa. Este es el caso de Morritos Alero 2.

El sitio constituye un alero formado por bloques de roca sedimentaria, que al derrumbarse longitudinalmente dejaron un espacio interno de apenas $0,8 \mathrm{~m}^{2}$ de superficie con alturas al techo que van desde $0,3 \mathrm{~m}$ hasta $1,5 \mathrm{~m}$ en el sector sur; $\mathrm{y}$ con una pequeña entrada natural de aproximadamente $0,4 \mathrm{~m}$ de largo por $0,3 \mathrm{~m}$ de ancho. El análisis de los materiales obtenidos en la excavación de esta unidad está en proceso. Esta evidencia incluye un conjunto poco diverso de puntas de proyectil triangulares pequeñas, cerámica y un astil completo, además de lascas y restos faunísticos; y está asociada con una fecha radiocarbónica, obtenida en una cuadrícula de sondeo exterior al alero de $810 \pm 40$ AP (AA66547) o 1189-1297 DC años calibrados ( $\mathrm{p}=95,4 \%$ ) con Oxcal 4, curva para el hemisferio sur (McCormac et al. 2004).

Un aspecto llamativo de Morritos Alero 2 es el uso que se les dio a las rocas naturales del alero como soporte para la ejecución de representaciones en donde predominan netamente 


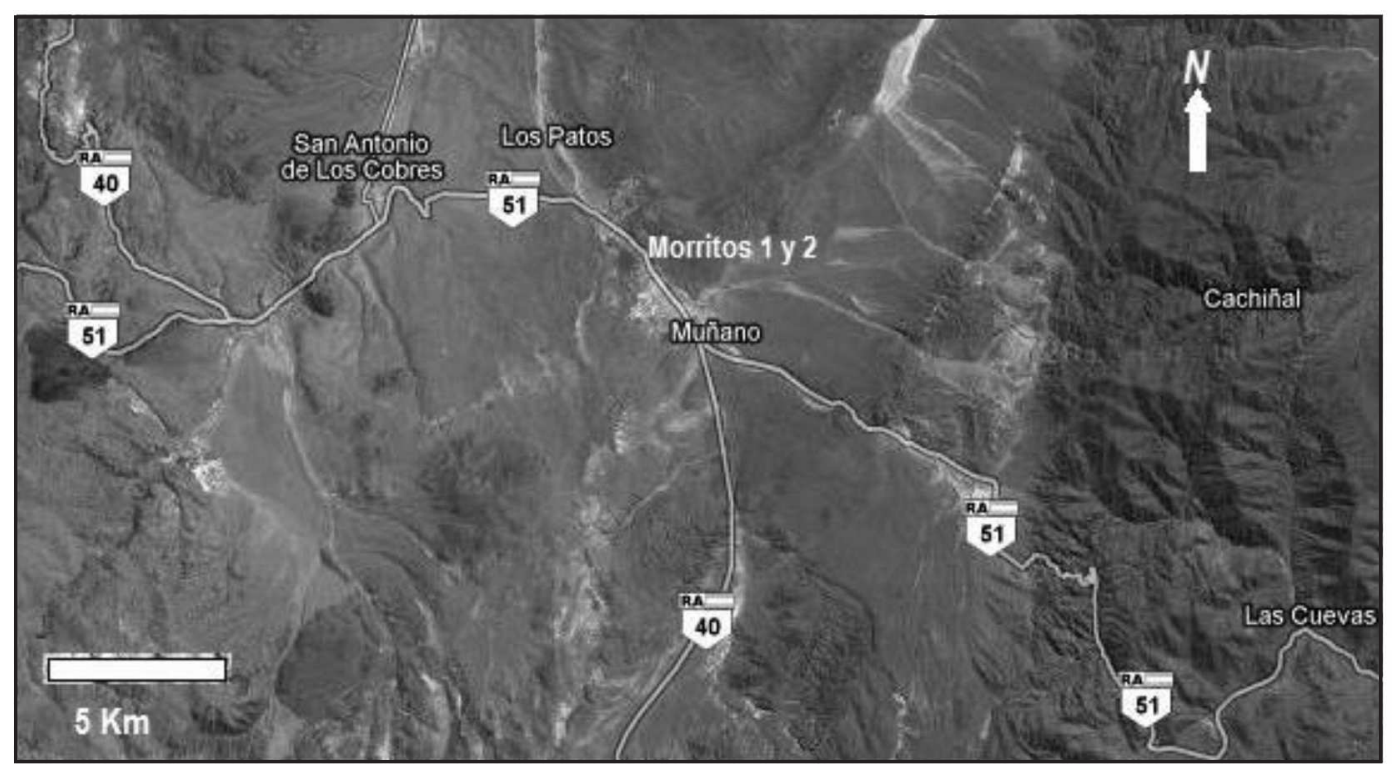

Figura 1: Ubicación de Morritos, puna de Salta, Argentina y su conexión con la Quebrada del Toro y Las Cuevas.

las figuras humanas con gran despliegue visual. Notoriamente en los paneles interiores localizados en el ala derecha de la pequeña entrada al alero (Figura 2,B y C). De este modo, la producción de las representaciones rupestres se hizo básicamente para ser vistas en el interior de un espacio pequeño y cerrado. Esto podría vincularse con un uso de este espacio cerrado para fines ceremoniales o como enterratorio, donde el énfasis, como veremos, se puso en la representación de figuras humanas con vestimentas y probables atributos de estatus.

\section{Descripción de las Representaciones Rupestres}

Las representaciones rupestres se disponen en tres paneles interiores y un panel exterior (Figura 2, A). En todos los casos se trata de petroglifos, mediante el grabado de figuras completas en donde el área extraída por el picado de la superficie natural de las rocas, que llega hasta los 0,3 mm de espesor, abarca el total de las figuras. Así la confección buscó resaltar el efecto figura- fondo. Brevemente, estas representaciones incluyen:

Camélidos: Hay dos representaciones restringidas al panel exterior, representando el 3,2 \% de un total 62 representaciones en todos los paneles. Ambas constituyen diseños de contornos irregulares cerrados de cuerpos anchos, colas pequeñas ligeramente levantadas y con cabezas esquematizadas dispuestas de frente presentando las dos orejas. Una de las representaciones, de cuerpo medianamente ancho y con dos patas, muestra cierto dinamismo por su inclinación hacia adelante (Figura 2,1). La otra representación presenta cuatro patas y una actitud estática (Figura 2,2). Estos diseños son comparables a los de otros sitios tardíos como Tastil y a los descriptos por Lanza (2000) para el valle Cachaquí Norte.

Pisadas: Están presentes tanto en el panel exterior como en los interiores del alero, con una frecuencia del $17,7 \%(n=62)$. Se trata de representaciones de pisadas humanas muy esquematizadas de contornos cerrados que abarcan desde figuras ovoidales (Figura 2,12) 
hasta un caso al cual se ha delineado el primer dedo (Figura 2,13). En el valle de SAC encontramos diseños comparables a este último desde el Período Temprano (Muscio 2006).

Figuras Antropomorfas: Son dominantes en los tres paneles, con una frecuencia total de $45 \%(n=62)$. Mayormente son representaciones escutiformes, con las características terminaciones agudas de los extremos de la figura. Estas representaciones están presentes en la cerámica Santa María Fases IV y V (ver como ejemplo la pieza 28 en Reynoso y Pratolongo 2008). Cuando están presentes, las piernas muestran cierto dinamismo por la flexión de una de ellas (Figura 2,5); o están bosquejadas separadas del cuerpo (Figura 2,4). Algunas de las representaciones antropomorfas incluyen la cabeza bosquejada como un triángulo invertido (Figura $2 \mathrm{C}$, superior). También hay casos en los cuales solo se ha bosquejado el cuello de forma rectangular, estando ausente la cabeza (Figuras 2,3; 1,6-7). En otros casos, estos escutiformes, presentan el cuello alargado y la cabeza con prolongaciones que sugieren tocados o adornos cefálicos (Figura 2,9). También hay casos que en la parte superior del cuerpo poseen prolongaciones, horizontales o levemente alzadas hacia arriba (Figura 2,10), a modo de manos bajo una vestimenta (poncho o capa). Además, hay un caso en el cual en el lado inferior izquierdo del cuerpo se ha bosquejado un apéndice rectangular, que podría tener por referente un lazo o atadura de vestimenta (Figura 2,5). Las vestimentas aparecen también adornadas, por ejemplo con triángulos (Figura 2,7). Un aspecto para resaltar es la inclusión de representaciones humanas pequeñas en los cuerpos de otras representaciones humanas de mucho mayor tamaño (Figura 2,7 y Figura 2,B superior). Un caso sugiere la decoración de una vasija o urna (Figura 2,8).

Otra clase de representaciones antropomorfas son las figuras con vestimentas cuadrangulares y lado ligeramente más ancho hacia los hombros (Figura 2,11). Estas pueden hacer referencia a uncus (Berenger et al 1985) o a petos de cuero (Achero 2000).

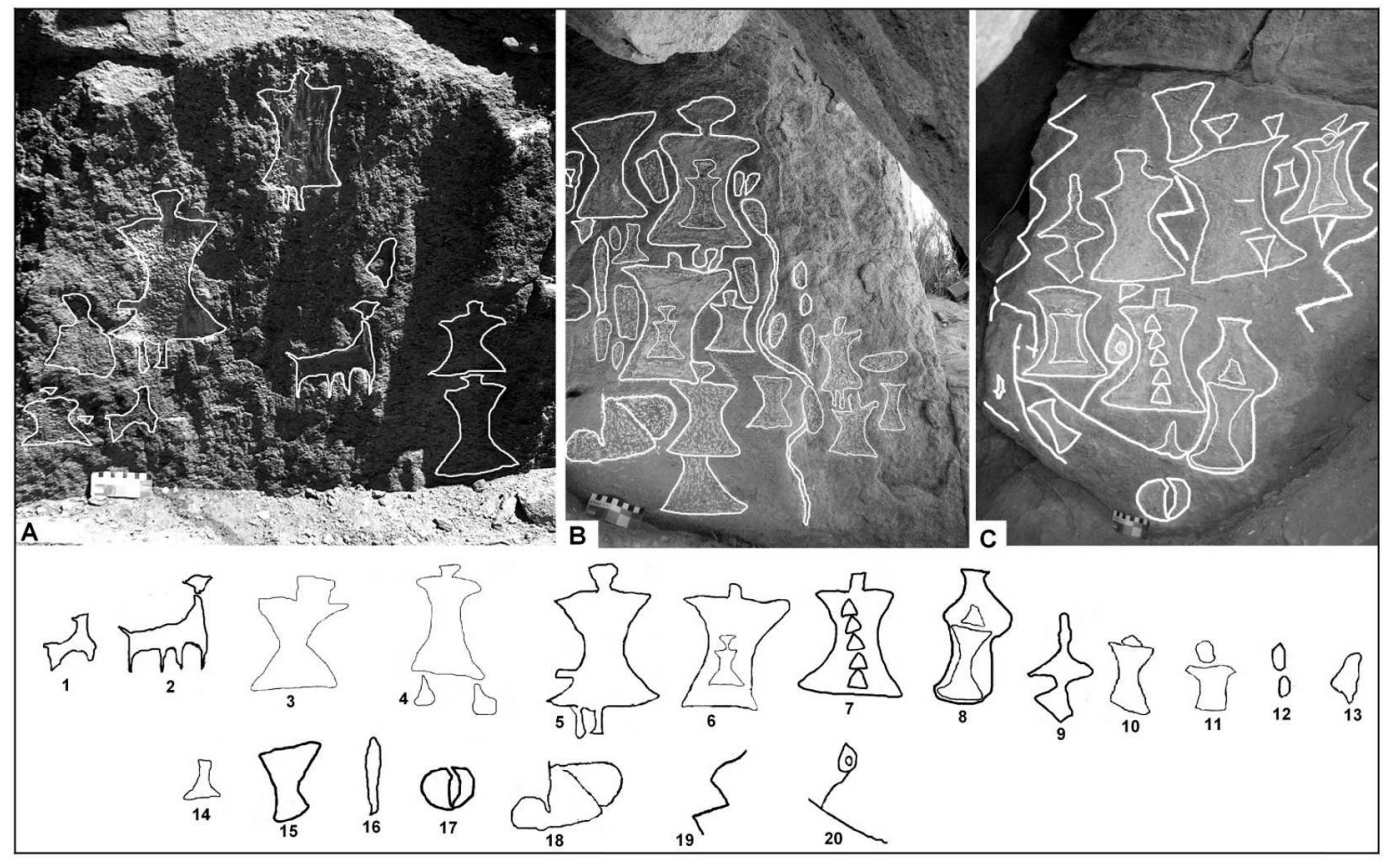

Figura 2: Paneles y representaciones Rupestres de Morritos Alero 2 
Otras Representaciones: Abarcan representaciones abstractas, como las líneas simples irregulares o líneas sinuosas, en zigzag, (Figura 2.19). Estas últimas se han utilizado para enmarcar lateralmente las escenas en los paneles (Figura 2,C). Las líneas irregulares se presentan asociadas a pisadas (Figura 2,B) o otras figuras semejantes a serpientes (Figura $2,20)$. Además, hay figuras circulares y curvilíneas (Figura 2,17; 1,18). Otros casos podrían remitir a hachas (Figuras 2,14; 1,15) o bastones (Figura 2,16).

\section{Discusión}

Las representaciones rupestres de Morritos Alero 2 se corresponden formalmente con la cronología radiocarbónica del Período Tardío. Los diseños escutiformes aparecen en otros contextos del NOA y del Norte de Chile en el Período Tardío y en la cerámica y en los discos de bronce Santamarianos. La evidencia arqueológica de Morritos y su localización en un entorno local en extremo árido, sugieren que la población asociada con la ocupación arqueológica debió tener un nicho económico con el pastoreo como estrategia predominante (Muscio 2009).

Los diseños de las representaciones rupestres de Morritos Alero 2, muestran que hacia el Período Tardío la población de la región del Valle de SAC participó de una sociedad de mayor escala; que compartió elementos simbólicos de amplia distribución espacial y que incluyó al Norte de Chile, a la puna y a los valles mesotermales del Noroeste de Argentina. Esta distribución espacialmente extensa de iconografía compartida debió responder a mecanismos sesgados de transmisión cultural (sensu Boyd y Richerson 1985), que actuaron en una escala espacial suprarregional preservando variación homóloga geográficamente muy extendida. El grado en el cual esta iconografía compartida registra variación local y las causas de esta variación, propician una línea de investigación futura. Lo importante aquí es resaltar que bajo la hipótesis planteada, la transmisión cultural no actuó acotando la replicación de los diseños rupestres en escalas espaciales pequeñas y por lo tanto fue en detrimento de la evolución de tradiciones locales diferenciadas. Esto último sí habría ocurrido durante el Período Temprano, a juzgar por la información de Matancillas (Muscio 2006).

El proceso de evolución cultural responsable de un patrón de distribución espacialmente extendido de iconografía debió responder a la emergencia de un nuevo entorno de aprendizaje, replicación y uso de esta iconografía. Aschero (2000) señala que la reducción de la diversidad regional con estandarización de representaciones rupestres durante el Tardío se basó en los cambios sociales relacionados con el surgimiento de jerarquías y con el aumento en la amplitud y distancia de las redes de tráfico caravanero. Ambas condiciones propician la emergencia de un nuevo entorno de aprendizaje social espacialmente amplio. La nueva situación social e ideológica del Tardío debió impartir los sesgos de transmisión, controlando el rango de variación cultural retenida, mediante criterios socialmente compartidos y basados, por ejemplo, en la territorialidad y el señalamiento de jerarquías que siguieron a la consolidación de las economías pastoriles en la puna. Por otra parte, la expansión espacial del tráfico caravanero debió incrementar la escala espacial de la transmisión por el aumento de las interacciones entre individuos de poblaciones locales distantes. Esto propició un entorno de aprendizaje favorecedor de una menor diferenciación de grupos en cuanto a los sesgos de transmisión y a los diseños iconográficos. Estas cuestiones requieren de investigación futura, documentando regionalmente las representaciones rupestres de la puna salteña, su diversidad y su historia filogenética. 


\section{Agradecimientos}

Este trabajo fue financiado por Conicet.

\section{Bibliografía Citada}

Aschero, C.

2000. Figuras humanas, camélidos y espacios en la interacción circumpuneña. En Arte en las rocas. Arte rupestre, menhires y piedras de colores en Argentina, M. Podestá y M. de Hoyos (Eds.), pp. 17-44. Editorial Sociedad Argentina de Antropología, Buenos Aires.

Berenguer, J.; V. Castro; C. Aldunante; C. Sinclaire y L. Cornejo 1985. Secuencia del arte rupestre del Alto Loa: una hipótesis de trabajo. Estudios en Arte Rupestre. Santiago. Museo Chileno de Arte Precolombino.

Boyd, R. y Richerson, P.

1985. Culture and the evolutionary process. University of Chicago Press, Chicago.

McCormac FG, AG Hogg, PG Blackwell, CE Buck, TFG Higham, and PJ Reimer. 2004. SHCal04 Southern Hemisphere Calibration 0 - 1000 cal BP Radiocarbon 46: 10871092.

Muscio, H. J.

2006. Aproximación Evolutiva a la Complejidad y al Orden Social Temprano a través del Estudio de Representaciones Rupestres de la Quebrada de Matancillas (Puna argentina). Estudios Atacameños N³1, pp. 9-30.

2009. Nicho y Estrategia Predominante. Dos Conceptos Útiles en Arqueología Evolutiva En 150 Años Después. La Vigencia de La Teoría Evolucionista de Charles Darwin, editado por M.C. Barboza; J. Davis Avila; C. Pícolli ; y J. Cornaglia Férnandez: 83-105. CEIA Universidad Nacional de Rosario, Rosario.

Lanza, M.

2000. Análisis estilístico del arte rupestre del valle calchaquí Norte, Salta. En Arte en las rocas. Arte rupestre, menhires y piedras de colores en Argentina, M. Podestá y M. de Hoyos (Eds.): 63-81. Editorial Sociedad Argentina de Antropología, Buenos Aires.

Reynoso, A y Pratolongo, G.

2008 Jaguares de nuevo. Consideraciones sobre la temática felínica en la iconografía cerámica del período Tardío en Yocavil (Noroeste Argentino). Estudios Atacameños: Arqueología y Antropología Surandinas N³5: 75-96. 
